\title{
Theoretical Calculation of Resonant Frequencies of the Human Alveolar Wall and Its Implications in Ultrasound Induced Lung Hemorrhage
}

\author{
D. John Jabaraj and Mohamad Suhaimi Jaafar
}

\begin{abstract}
The human alveolar wall was modeled here as membranes and its resonant frequency was determined in order to analyze the mechanism of alveolar resonance of the ultrasound induced lung hemorrhage. The resonant frequency of the membrane models of the human alveolar wall was determined to be within the range of the frequency of diagnostic ultrasound used in the thoracic and abdominal regions; which is $\geq 2.5 \mathrm{MHz}$. Thus, the alveolar resonance is proved to occur during the diagnostic ultrasound imaging of humans. However the possibility of alveolar wall damage at resonance was not analyzed here. Nevertheless according to the resonant frequency equations derived here, the resonance of the alveolar wall by diagnostic ultrasound waves can be eliminated from occurring when total lung capacity is $\sim 20 \%$ (at maximal expiration).
\end{abstract}

Index Terms-Alveolar resonance, lung hemorrhage, resonant frequency, ultrasound.

\section{INTRODUCTION}

The ultrasound (US) is widely used in the medical field as an indispensable tool for non-invasive diagnostic imaging and therapy. When compared to other medical imaging procedures, the clinical use of the diagnostic US has had good safety record with no documented cases of adverse effects on humans [1]. The historical truth is that the US was thought to be relatively safe until the year 1951, when researchers developing the diagnostic US equipments, became aware of the potential risk to tissues and organs [2].

The frequency of US normally used for diagnostic imaging purposes is in the range of $1-10 \mathrm{MHz}$ depending on the procedures [3]. Nevertheless, the US at diagnostic imaging level too can be shown to cause variety of bio-effects in tissue (in vitro and in vivo) depending on the exposure conditions and tissue structures. Particularly in the lungs, the US interaction is seen to be intensified because the lungs are such delicate structure with many air interfaces with tissue. The US induced lung damage has been observed in animal experimentations at the exposure levels of US examinations for humans. Lung lesions due to pulsed US have been seen to occur in mice, rats, rabbits, apes and pigs [4] - [7]. This US induced damage to the lung's microvasculature is

Manuscript received October 9, 2012; revised November 29, 2012. This work was supported in part by the USM PGRS Grant and the UniKL Financial Assistance Scheme.

D. John Jabaraj is with the MSI-UniKL Kedah and the Dept. of Medical Physics, Universiti Sains Malaysia, 11800 USM, Penang, Malaysia (e-mail: jojab77@yahoo.com).

Mohamad Suhaimi Jaafar is with the Dept. of Medical Physics, Universiti Sains Malaysia, 11800 USM, Penang, Malaysia (e-mail: msj@usm.my). characterized by localized extravasations of red blood cells from capillaries into the alveolar space [8].

Both thermal and mechanical mechanisms have been proposed as to understand what the cause of US induced lung hemorrhage is. Nevertheless, the thermal mechanism (heating effect) has been ruled out as there are evidences of it failing to explain lung hemorrhage. Heating effect of US waves resulting in lung tissue damage, have been experimentally excluded by using thermocouple measurements [9] and by using pathological analyzation of laser induced lesion [10].

Particularly, the mechanical mechanism known as inertial cavitations have been widely researched. Inertial cavitations is the bursting of the micro-bubbles through resonance induced by US waves, causing high energy emanations that damages surrounding tissues [11]. Although gas in tissue is present as in the alveoli, the theory of acoustic inertial cavitations as the US damage mechanism in the lungs is not supported by some studies [12]. There is evidence of increased hydrostatic pressure increasing lung damage, which is the opposite of the inertial cavitations prediction [13].

There is another mechanical mechanism known as the alveolar resonance which might explain the US induced lung hemorrhage. The mechanism suggests that US propagation in lungs results in alveolar response to compression and tensional waves [14]. This deformation might damage the components within alveolar wall. However, there are no mathematical calculations or experimental data or models that support or disprove the mechanism of alveolar resonance in producing US induced lung hemorrhage.

\section{THEORY}

\section{A. The Alveolus Structure}

An alveolus is actually not spherical but is in fact polygonal [15]. The alveolus is shaped more alike a multifaceted polygon with almost flat surface walls and can be represented by a dodecahedron as in Fig. 1 [16].

Within the alveolar wall, the capillaries are arranged as a single layer separated from air spaces by a thin cellular barrier called the blood gas barrier (BGB). The BGB is relatively very thin (about $200 \mathrm{~nm}$ ) and due to its inability to expand under oscillation, it is susceptible to rupture [17].

The elastic tension of the alveolar wall depends on the strain caused by normal tidal breathing. During inspiration and expiration of the normal breath, the alveolus undergoes major changes in dimension only between $0 \%$ to $20 \%$ of Total Lung Capacity (TLC) [18]. However the resulting strain from 
$0 \%$ to $20 \%$ TLC is about zero because the walls are not stretched but are just unfolded (recruitment). From 20\% TLC onwards (after recruitment), the alveolus size changes only slightly from $0-0.05$ linear strain during normal tidal breathing [19]. This translate to areal strain of $0-0.1025$ (Appendix A).

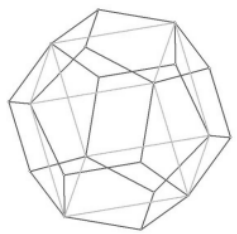

Fig. 1. The alveolus represented as a dodecahedron.

\section{B. The Two Dimensional Wave Equation}

The two dimensional wave equation is formulated by focusing on an element in the membrane. The Fig. 2 shows an arbitrary element $d x$ - $d y$ of membrane and the tension forces, $T$ acting on it while displacement is $z$ at time, $t$.

The two dimensional wave's equation is [21];

$$
\partial^{2} z / \partial t^{2}=c_{T}^{2}\left(\left[\partial^{2} z / \partial x^{2}\right]+\left[\partial^{2} z / \partial y^{2}\right]\right)
$$

With transverse wave velocity;

$$
c_{T}=(T / \sigma)^{1 / 2} .
$$

The area density, $\sigma$ is mass over area. The best coordinate system must be selected for a membrane shape so that the wave equation can be solved to obtain the resonant frequency equation.

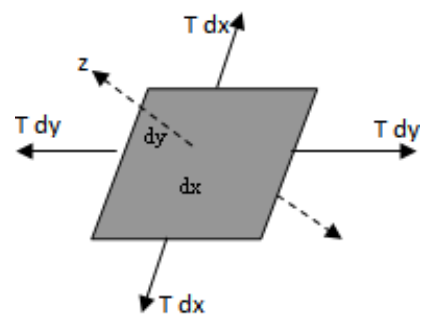

Fig. 2. The axes $(x, y, z)$ and forces $(T d x, T d y)$ on a membrane element with displacement $z$

\section{Methodology}

The very first step taken in this study of the alveolar resonance is to examine and simplify the alveolar wall into structural model. Certain assumptions were made for the modeling purposes. The alveolar wall is assumed as homogenous, isotropic and linearly elastic clamped membrane.

Next, the theory of vibration was applied to the alveolar wall model to determine the resonant frequency equation. Then, the risks of US induced lung hemorrhage was analyzed and predicted based on this study. Preventive measures concerning US induced lung hemorrhage were also determined.

The facets of the alveolar structure are shaped as flat irregular polygons that are made of the thin alveolar wall [15]. For the simplification in modeling purposes, it is thus proposed here for the alveolar wall facets to be reduced as regular polygons.

The application of the theory of vibration on membrane has a certain restriction. A suitable coordinate system is needed in order to easily determine the boundary condition of the membrane [20]. This is to ensure that the resulting partial differential equation (PDE) arising from the application of vibration theory on the membrane, is not extremely complicated and is not only partially or approximately solvable [21].

The simple Cartesian coordinate system fits the boundaries of the square or rectangular four-sided polygon. Thus for simplification purposes, the shape of the alveolar wall facet is proposed and modeled here as a four-sided square membrane as shown in Fig. 3.

Resonant frequency of square membrane with side-length, $L$ at $n_{x}$ and $n_{y}$ mode is [21];

$$
f_{n x}, n_{y}=1 / 2 c_{T} L^{-1}\left[n_{x}^{2}+n y^{2}\right]^{1 / 2}
$$

Likewise, the polar coordinate system fits the boundaries of the circular shape, and so the resulting PDE from the application of vibration theory can also be solved completely. Thus for simplification purposes, the shape of the alveolar wall facet is also proposed and modeled here as a circular membrane too (Fig. 4).

Resonant frequency of circular membrane with radius, $r$ at $n$ mode is [21];

$$
f_{n}=1 / 2 c_{T} r^{-1}[n-1 / 4]
$$

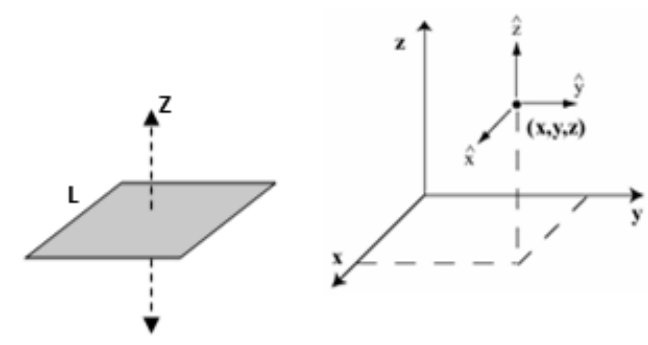

Fig. 3. The Cartesian coordinates for the square membrane.
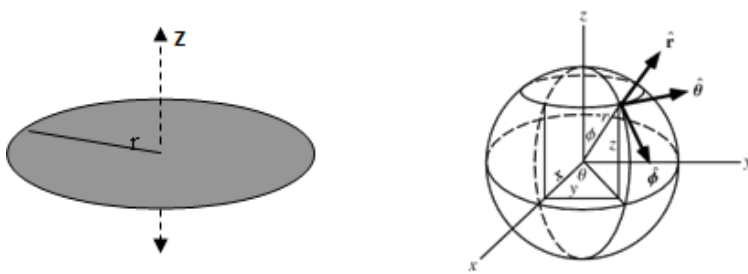

Fig. 4. The polar coordinates for the circular membrane.

\section{RESUlTS AND DISCUSSION}

The resonant frequency of the membrane models of the alveolar wall basically depends on the transverse wave velocity in membrane, the dimension of the alveolar wall (side-length or radius) and the vibration modes.

\section{A. Transverse Wave Velocity}

The tension, $T$ of the membrane is primarily the elastic tension and is dependent on the membranes' Young's modulus, $E$; the areal strain of tidal breath, $\varepsilon_{b}$ and the membrane thickness, $d$ (Appendix B). 


$$
T=E \varepsilon d
$$

The Young's modulus equation can be determined by utilizing its relationship with the bulk modulus, $K$. The Poisson ratio of material is denoted as $u$ while $\rho$ is the membranes' density and $c_{L}$ is the longitudinal wave speed.

$$
\begin{gathered}
E=3 K(1-2 u) \\
E=3\left[\rho c_{L}^{2}\right](1-2 u)
\end{gathered}
$$

A relationship between wave velocities and the areal strain of tidal breath for the membrane models of the alveolar wall was derived through applying (5) and (7) into (2);

$$
c_{T}=(3-6 u)^{1 / 2} c_{L} \varepsilon b^{1 / 2}
$$

The Poisson's ratio for alveolar wall have the value ranging from $0.35-0.45$ [22]. The longitudinal speed of sound wave in soft tissues ranges from $1490 \mathrm{~m} / \mathrm{s}-1610 \mathrm{~m} / \mathrm{s}$ at $37^{\circ} \mathrm{C}$ body temperature [23]. Whenever calculations involve ranges, the interval arithmetic method should be used. Here the alveolar wall is modeled as membranes and the substitution of alveolar wall's characteristic interval values into (8) results in;

$$
c_{T}=\{816.11,1527.38\} \varepsilon b^{1 / 2} \mathrm{~m} / \mathrm{s}
$$

\section{B. The Resonant Frequency of Square Membrane Model}

The resonant frequency equation of the square membrane model of the alveolar wall was analyzed by using the human alveolus. The radius of human alveolus ranges from 100 - 200 $\mu \mathrm{m}$ [24]. If the facets of the alveolar wall are modeled as a square shaped membrane, then the length dimension is the side-length which can be estimated as twice the radius. Thus the side-length in interval notation is $\{200,400\} \mu \mathrm{m}$.

The fundamental frequency occur when the mode is at $n_{x}=$ $n_{y}=1$. If the side-length range and (9) are substituted into (3), then the fundamental frequency equation (in vacuum) for the square membrane model of human alveolar wall will be;

$$
f_{1,1}=\{1.443,5.400\} \varepsilon b^{1 / 2} M H z
$$

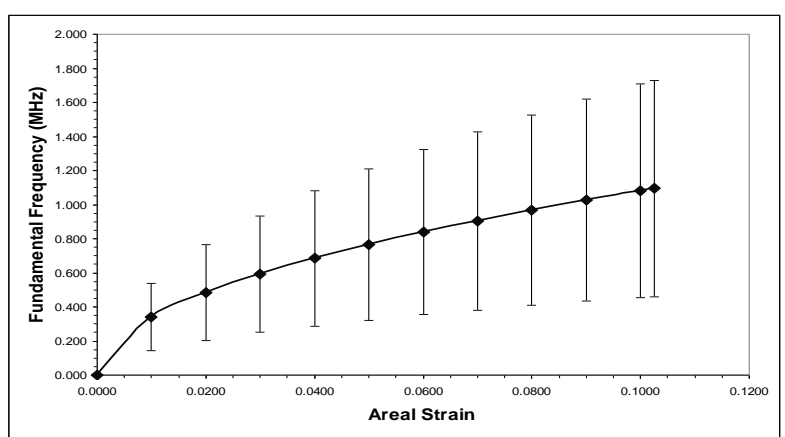

Fig. 5. The ranges of fundamental frequency vs. areal strain of tidal breath with upper limit for $200 \mu \mathrm{m}$ side-lengths and lower limit for $400 \mu \mathrm{m}$ side-lengths of the modeled square membrane of the human alveolar wall.

The relationship between the fundamental frequencies of the square membrane model of the human alveolar wall, and the areal strain due to tidal breath can be analyzed in Fig. 5.
The fundamental frequency exceeds $1 \mathrm{MHz}$ when the tidal breath areal strain is higher than 0.0343 to until its limit at 0.1025 . At the maximum tidal breath areal strain of 0.1025 , the maximum fundamental frequency is determined to be $1.729 \mathrm{MHz}$.

For illustrative purposes, the modes frequencies for the square membrane model when transverse wave velocity is $100 \mathrm{~m} / \mathrm{s}$ and the mean alveolar side-length dimension in human is $300 \mu \mathrm{m}$, is calculated and then depicted in Fig. 6.

$$
f_{n x}, n_{y}=0.1667\left[n_{x}^{2}+n_{y}{ }^{2}\right]^{1 / 2} \quad \mathrm{MHz}
$$

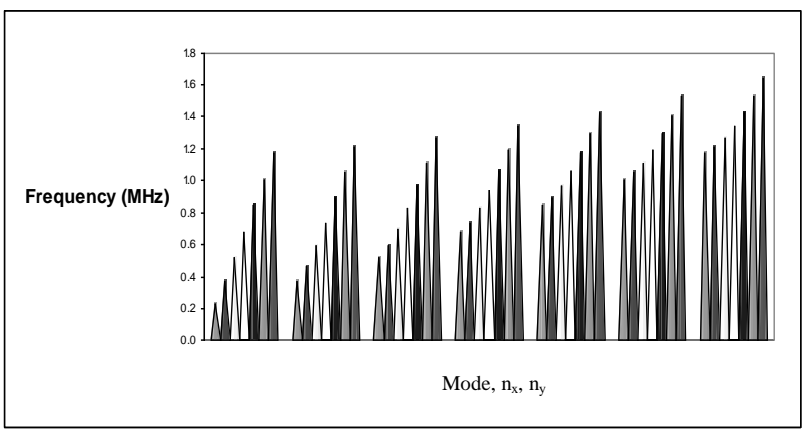

Fig. 6. The resonant frequencies mode values (in vacuum) of the square membrane model of the human alveolar wall at $100 \mathrm{~m} / \mathrm{s}$ transverse wave for $300 \mu \mathrm{m}$ side-length.

\section{The Resonant Frequency of Circular Membrane Model}

The resonant frequency equation of the circular membrane model of the alveolar wall was studied by using the human alveolus. If the membrane model surface is considered as circular, then the length dimension is the radius $\left(r_{\mathrm{o}}\right)$ equivalent to the human alveolus radius of $\{100,200\} \mu \mathrm{m}$.

The fundamental frequency occur when the mode is at $n=1$. Thus the fundamental frequency equation (in vacuum) for the circular membrane model of the human alveolar wall was derived by substituting (9) and the alveolar radius range into (4).

$$
f_{1}=\{1.530,5.728\} \varepsilon b^{1 / 2} \quad \mathrm{MHz}
$$

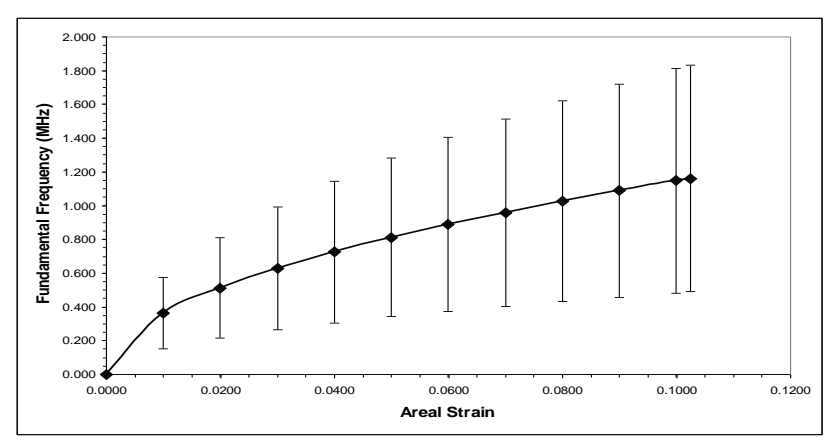

Fig. 7. The relation between fundamental frequency ranges with membrane areal strain of tidal breath for the modelled circular membrane of the human alveolar wall with upper limit for $100 \mu \mathrm{m}$ and lower limit for $200 \mu \mathrm{m}$ radius.

The relationship between the fundamental frequencies of human alveolar wall (when modeled as a circular membrane) and the areal strain of tidal breath can be analyzed in Fig. 7. The fundamental frequency exceeds $1 \mathrm{MHz}$ at areal strain of tidal breath value of 0.0305 to till the maximum of 0.1025 . At maximum tidal breath areal strain of 0.1025 , the maximum fundamental frequency is $1.834 \mathrm{MHz}$. 
For illustrative purposes, the modes resonant frequencies for the circular membrane model of the alveolar wall when transverse wave velocity is $100 \mathrm{~m} / \mathrm{s}$ for the $150 \mu \mathrm{m}$ mean radius human alveolar wall, is depicted in Fig. 8.

$$
f_{n}=0.3333[n-1 / 4] \quad \mathrm{MHz}
$$

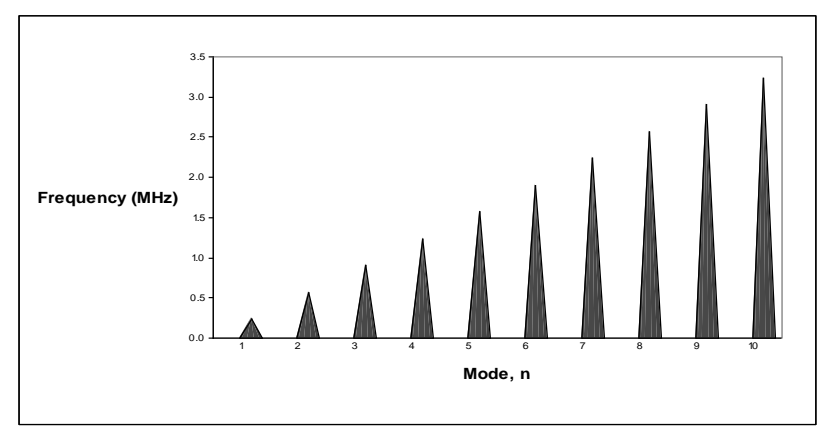

Fig. 8. The mode resonant frequencies values of the circular membrane model of the human alveolar wall when transverse wave velocity is $100 \mathrm{~m} / \mathrm{s}$ for $150 \mu \mathrm{m}$ mean radius.

\section{DISCUSSION}

It is considered that if the US frequency matches the resonant frequencies of membranes models, then resonance occurs in alveolar wall resulting in possible damage (depending on US pressure). The frequency normally used in diagnostic US is $\geq 1 \mathrm{MHz}$. However for the imaging of the organs in the thoracic and abdominal regions, the US of $\geq 2.5$ $\mathrm{MHz}$ is utilized [25]. Hence the lungs are normally exposed to US frequencies of $\geq 2.5 \mathrm{MHz}$.

From the calculations of this study, the fundamental frequencies of the human alveolar wall (when modeled as square and circular vibrating membranes) have the maximum values of $1.729 \mathrm{MHz}$ and $1.834 \mathrm{MHz}$ respectively. Nevertheless, the higher modes resonant frequencies which are multiples of the fundamental frequency can be $\geq 2.5 \mathrm{MHz}$ (within the diagnostic US frequency range) as depicted in Fig. 7 and 8. This means there is a possibility for the alveolar structure in humans to be affected by diagnostic US exposure through higher mode resonance only. Thus it is proven here that the alveolar structure is not too large to oscillate and to be affected in regard of the diagnostic US frequency range as stated by some studies [26].

The resonant frequency of the alveolar wall (as according to the membrane models) does depend on the transverse wave velocity which can have the value $0-489 \mathrm{~m} / \mathrm{s}$ depending on the areal strain of tidal breath from $0-0.1025$.

The resonant frequency can be made non-existent $(0 \mathrm{MHz})$ if the transverse wave velocity in the membrane is also $0 \mathrm{~m} / \mathrm{s}$. This occurs when areal strain of tidal breath is $\sim 0$ when lungs' volume is under $20 \%$ TLC (maximal expiration). Thus according to this study, it is possible to avoid the US induced lung hemorrhage by keeping the TLC 20\% during US exposure in order for the alveolar wall to not have any resonant frequency and so in any way to be affected. This could be recommended (based on this study) as a preventive measure to avoid US induced lung hemorrhage from occurring in humans.

The alveolar wall was modeled as a two dimensional vibrating membrane instead of as vibrating plate because the stiffness value was deemed to be too small and approaching zero [27]. It was assumed that the membrane models had fixed boundary (clamped) as the alveolar wall is supported by other alveolar walls at the edge. The alveolar wall was further assumed as being homogenous because it is continuous, isotropic because it is directionally independent to stress and linearly elastic because it contains elastin fibers and the strain is small.

\section{APPENDIX}

\section{A. Areal Strain of Tidal Breath}

The relation between areal strain and linear strain obtained from [29];

$$
\alpha=(\varepsilon+1)^{1 / 2}-1
$$

During normal tidal breathing, the linear strain changes between $0 \leq \alpha \leq 0.05$ [19] and so the areal strain changes;

$$
\begin{aligned}
& 0 \leq \alpha \leq 0.05 \\
& 0 \leq\left[(\varepsilon+1)^{1 / 2}-1\right] \leq 0.05 \\
& 0 \leq \varepsilon \leq 0.1025
\end{aligned}
$$

\section{B. The Formulae of Tension in Membrane}

Assume that an arbitrary piece of membrane which at zero external stress has an area, $A_{0}$, is stretched to a size $A_{1}>A_{0}$ as seen in Fig. 9. The energy change at lowest form is;

$$
U_{\text {stretch }}=1 / 2 k_{\text {stretch }}\left(A_{1}-A_{0}\right)^{2} / A_{0}
$$

where the modulus $k_{\text {stretch }}$ is the proportionality constant between a quadratic deviation of the area from its unstressed state and the respective energy. The additional $A_{0}^{-1}$ is a physics convention [28].

The tension under which the membrane is subjected to, is the derivative of energy with respect to area;

$$
\begin{aligned}
T & =\partial U_{\text {strecth }} / \partial A_{1} \\
& =k_{\text {strecth }}\left(A_{1}-A_{0}\right) / A_{0} \\
& =k_{\text {streckh }} \varepsilon \\
& =E d \varepsilon
\end{aligned}
$$

The defined dimensionless areal strain is, $\varepsilon=\left(A_{1}-A_{0}\right) / A_{0}$. The constant of proportionality is, $k_{\text {stretch }}=E d$, where $E$ is Young's modulus and $d$ is membrane thickness. The membrane tension equation follows the Hooke's Law for membrane stretching, where stress is proportional to strain and to the constant of proportionality $\mathrm{k}_{\text {stretch }}$.

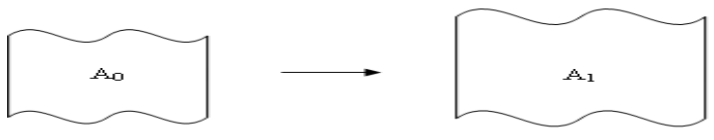

Fig. 9. The deformation of an area

\section{ACKNOWLEDGMENT}

D. John Jabaraj thanks Mrs. Periathai Darling for many things and for always being a great amma. 


\section{REFERENCES}

[1] T. L. Szabo, Diagnostic Ultrasound Imaging: Inside Out, 1st ed. San Diego, USA: Academic Press, 2004, pp. 511.

[2] L. A. French, J. J. Wild, and D. Neal, "Attempts to determine harmful effects of pulsed ultrasonic vibrations," Cancer, vol. 4, pp. 342-344, 1951.

[3] D. Dalecki, "Mechanical bioeffects of ultrasound," Annu. Rev. Biomed. Eng., vol. 6, pp. 229-48, 2004.

[4] S. Z. Child, C. L. Hartman, L. A. Schery, and E. L. Carstensen, "Lung damage from exposure to pulsed ultrasound," Ultrasound Med. Biol. , vol. 16, pp. 817-825, 1990

[5] A. F. Tarantal and D. R. Canfield, "Ultrasound-induced lung hemorrhage in the monkey," Ultrasound Med Biol., vol. 20, no. 1, pp. 65-72, 1994.

[6] R. Baggs, D. P. Penney, C. Cox, S. Z. Child, C. H. Raeman, D. Dalecki, and E. L. Carstensen, "Thresholds for ultrasonically induced lung hemorrhage in neonatal swine," Ultrasound Med Biol., vol. 22, pp. 119-128, 1996.

[7] D. Dalecki, S. Z. Child, C. H. Raeman, C. Cox, and E. L Carstensen, "Ultrasonically induced lung hemorrhage in young swine," Ultrasound Med Biol., vol. 23, pp. 777-81, 1997.

[8] D. P. Penney, E. A. Schenk, K. Maltby, C. Hartman-Raeman, S. Z Child, E. L. Carstensen, "Morphologic effects pf pulsed ultrasound in the lungs," Ultrasound Med. Biol., vol.19, pp.127-135, 1993.

[9] C. L. Hartman, S. Z. Child, D. P. Penney, and E. L. Carstensen, "Ultrasonic heating of lung tissue," J. Acoust. Soc. Am., vol. 91, pp. 513-516, 1992.

[10] J. F. Zachery, J. P. Blue, R. J. Miller, B. J. Ricconi, J. G. Eden, and W. D. Jr. O'Brien, "Lesions of ultrasound -induced lung hemorrhage are not consistent with thermal injury," Ultrasound Med. Biol., vol. 32, no. 11, pp.1763-1770, Nov 2006.

[11] N. Kudo and K. Yamamoto, "Impact of bubbles on ultrasound safety," International Congress Series, vol. 1274, pp. 129-132, 2004.

[12] E. L. Carstensen, S. Gracewski, and D. Dalecki, "The search for cavitation in vivo," Ultrasound Med. Biol., vol. 26, pp.1377-1385, 2000.

[13] W. D. Jr. O’Brien, L. A. Frizzell, and M. Weigel, “Ultrasound-induced lung haemorrhage is not caused by inertial cavitation," J. Acoust. Soc. Am., vol. 108, pp. 1290-1297, 2000.

[14] C. C. Church, E. L. Carstensen, W. L. Nyborg, P. L. Carson, L. A. Frizzell, M. R. Balley, "The risk of exposure to diagnostic ultrasound in post-natal subjects-nonthermal mechanisms," J. Ultrasound Med., vol. 27, pp. 565-592, 2008.
[15] H. D. Prange, "Laplace's law and the alveolus: a misconception of anatomy and a misapplication of physics," Adv Physiol Educ., vol. 27, no. $1-4$, pp. 34-40, Dec 2003.

[16] A. D. Karakaplan, "A mathematical model of lung parenchyma," $J$ Biomech. Eng., vol. 102, no. 2, pp.102:124, May 1980.

[17] J. N. Maina and J. B. West, "Thin and Strong! The Bioengineering Dilemma in the Structural and Functional Design of the Blood-Gas Barrier," School Physiol. Rev., vol. 85, pp. 811-844, 2005.

[18] D. E. Carney, C. E. Bredenberg, H. J. Schiller, A. L. Picone, U. G. McCann II, L. A. Gatto, G. Bailey, M. Fillinger, and G. F. Nieman, "The Mechanism of Lung Volume Change during Mechanical Ventilation,” Am. J. Respir. Crit. Care Med., vol. 160, pp. 1697-1702, 1999.

[19] E. Roan and C. M. Waters, "What do we know about mechanical strain in lung alveoli?," Am. J. Physiol. Lung Cell Mol. Physiol.,vol. 301, pp. L625-L635, 2011

[20] G. Chen, M. P. Coleman and J. Zhou, "Analysis of Vibration Eigenfrequencies of a Thin Plate by the Keller-Rubinow Wave Method I: Clamped Boundary Conditions with Rectangular or circular geometry," SIAM Journal on Applied Mathematics, vol. 51, no. 4, aug., 1991.

[21] W. C. Elmore and M. A. Heald, "Physics of Waves," Dover Publications Inc, 1985

[22] S. J. Lai-Fook, T.A. Wilson, R. E. Hyatt, J. R. Rodarte, "Elastic constants of inflated lobes of dog lungs," J. Appl. Physiol., vol. 40, no. 4, pp.508-13, Apr 1976.

[23] G. D. Ludwig, "The Velocity of Sound through Tissues and the Acoustic Impedance of Tissues," J. Accous. Soc. Am., vol. 22, no. 6, pp. 862-66, Nov 1950

[24] P. M. Treuting and S. M. Dintzis, Comparative Anatomy and Histology: A Mouse and Human Atlas, Academic Press, 1 edition December 30, 2011.

[25] R. J. Fitzgibbons and A. G. Greenburg, Nyhus and Condon's Hernia, Lippincott Williams \& Wilkins, Fifth edition, Nov 15, 2001.

[26] G. Ter Haar, "Ultrasound bioeffects and safety," in Proc. Inst. Mech. Eng., vol. 224, no. 2, pp. 363-73, 2010.

[27] R. Vepa, Dynamics of Smart Structures, John Wiley \& Sons, 2010, pp. 178.

[28] M. Deserno, "Membrane Elasticity and Mediated Interactions in Continuum Theory: A Differential Geometric Approach," Handbook of Modern Biophysics, Springer-Humana Press, 2009, vol. 2, chap.2, pp. 101-120.

[29] A. Gefen, D. Elad, and R. J. Shiner, "Analysis of stress distribution in the alveolar septa of normal and simulated emphysematic lungs," $J$. Biomech., vol. 32, no. 9, pp.891-7, Sept. 1999. 